\title{
Polychromatic Artistic Components as Interior Decorative Elements from the Constanta Casino Monument ${ }^{+}$
}

\author{
Ionut Marina ${ }^{1, *}$, Sofia Teodorescu ${ }^{2}$ and Rodica-Mariana Ion ${ }^{1,2}$ \\ 1 Doctoral School of Materials Engineering, Valahia University of Targoviste, 130004 Târgoviște, Romania; \\ rodica.ion@icechim.ro \\ 2 National Institute for Research and Development in Chemistry and Petrochemistry-ICECHIM, \\ 202 Spl. Independenței, 060021 Bucharest, Romania; sofiateodorescu@icstm.ro \\ * Correspondence: ionutmarina.office@gmail.com \\ + Presented at the 16th International Symposium "Priorities of Chemistry for a Sustainable Development" \\ PRIOCHEM, Bucharest, Romania, 28-30 October 2020.
}

Published: 16 November 2020

Keywords: consolidants; Casino; FTIR; composition

The Casino building from Constanta is the most important and characteristic public building built in the Art Nouveau style [1]. The stylistic unity of the treatment of the elements is remarkable: the volume, the façades, the shape of the gaps, the modernization, the interior and exterior color decoration (stuccoes), the furniture elements of the interior, and the exploitation of the fluidity of the location [2]. The building was designed as a parallelepiped mounted horizontally which gives the impression of stability, but in which each of the five visible sides (four façades and roof), are different from spatial, volumetric, and decorative points of view. Besides the elements of the artistic components described above, there are some decorative elements from the repertoire of the Art Nouveau style: floral reliefs, curved decorative elements in spirals or waves, painted ceilings, railing and window hardware, lighting fixtures, and stained glass (which unfortunately only retains traces of the original ones) all contribute to the harmony of the general composition. In this paper, some compositional aspects of the pigments, collected from the Theater Hall of the Casino's stuccoes, have been investigated and discussed.

Fourier-transform infrared (FTIR) spectrometry was used to identify functional groups of the analyzed materials. FTIR spectrometry was performed with single-bounce diamond-attenuated total reflectance (ATR) using a Vertex 80v spectrometer (Bruker Optik GMBH, Ettlingen, Germany), equipped with a Diffuse Reflectance Infrared Fourier Transform (DRIFT) accessory in the range of $4000-400 \mathrm{~cm}^{-1}$.

FTIR spectra of the analyzed samples presents several peaks comparable to those in the literature $[2,3]$. The broad band located in the region $3300-3500 \mathrm{~cm}^{-1}$ arises from the stretching vibration of the hydroxyl groups of the layers, as well as the water molecules from the humidity. The band at 1632 $\mathrm{cm}^{-1}$ is attributed to the bending vibration of water $(\mathrm{vH}-\mathrm{O}-\mathrm{H})$; a band at $1410 \mathrm{~cm}^{-1}$ was also observed, implying a contribution of bicarbonates in the interlayer space. Bands lower than $1000 \mathrm{~cm}^{-1}$ could be assigned to the vibration mode of calcium carbonate $\left(875\right.$ and $\left.712 \mathrm{~cm}^{-1}\right)$ and silica $\left(798\right.$ and $\left.779 \mathrm{~cm}^{-1}\right)$ and possibly iron oxides $\left(570 \mathrm{~cm}^{-1}\right)$ for pink color.

The composition identified by the FTIR technique is extremely important for the restoration works: a mixture of calcite, silica, and iron oxides. 


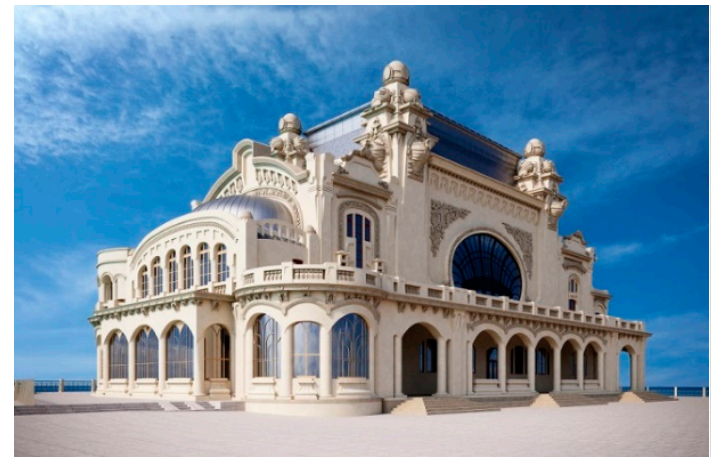

(a)

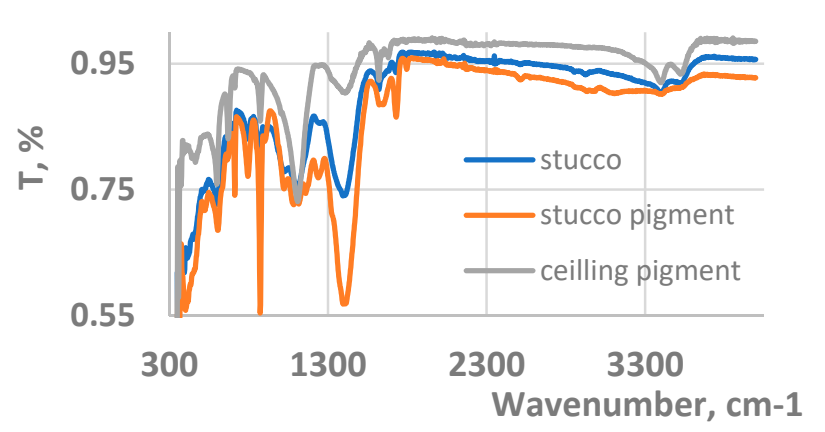

(b)

Figure 1. Casino Building (a) and FTIR spectra of some stuccoes from the Theater Hall of the Casino (b).

\section{References}

1. Marina, I.; Ion, R.M.; David, M.E.; Iancu, L.; Grigorescu, R.M. Investigation of Chromatic Parameters of Some Samples from Constanta Casino. Multidiscip. Digit. Publ. Inst. Proc. 2019, 29, 64, doi:10.3390/proceedings2019029064.

2. Ion, R.M.; Iancu, L.; David, M.E.; Grigorescu, R.M.; Trica, B.; Somoghi, R.; Vasile, S.F.; Dulama, I.D.; Gheboianu, A.I.; Tincu, S. Multi-Analytical Characterization of Corvins' Castle-Deserted Tower. Construction Materials and Conservation Tests. Heritage 2020, 3, 941-964, doi:10.3390/heritage3030051.

3. Iancu, L.; Ion, R.M.; Grigorescu, R.M.; David, M.E.; Ghiurea, M.; Vasilievici, G.; Stirbescu, R.M.; Dulama, I.D. Double substituted carbonated hydroxyapatite for stone consolidation. J. Sci. Arts 2020, 3, 713-730, doi:10.46939/J.Sci.Arts-20.3-b03.

Publisher's Note: MDPI stays neutral regarding jurisdictional claims in published maps and institutional affiliations.

(C) 2020 by the authors. Licensee MDPI, Basel, Switzerland. This article is an open access article distributed under the terms and conditions of the Creative Commons Attribution (CC BY) license (http://creativecommons.org/licenses/by/4.0/). 